\title{
Reprocessamento de produtos médicos: da política regulatória à prática operacional
}

\author{
The reprocessing of medical products: \\ from regulatory polices to operational practices
}

Eliana Auxiliadora Magalhães Costa ${ }^{1}$

Ediná Alves Costa ${ }^{1}$

${ }^{1}$ Instituto de Saúde Coletiva, Universidade Federal da Bahia. Rua Augusto Viana s/n, Canela. 40.110-060 Salvador BA. costaeliana2003@hotmail.com

\begin{abstract}
The number of technological resources used in health care interventions is growing and continually expanding with the introduction of new products and articles. Problems associated with the reutilization of medical products, both reusable and of single use, affect policies and related technical-operational, economic, political, ethical, legal, and environmental matters. This study aims to contextualize the regulatory systems of medical products, and analyze the subsequent operational implications for Brazilian hospital practices. The article consists of a bibliographic review, carried out without time and language restriction, utilizing the Web of Science, Bireme, Scielo and Lilacs databases, with the support of specific descriptors. This study uses the contextualization of regulatory plans for medical products across the world and in Brazil and the existing condition of standardization of the reprocessing of these products as the assessment sources with which to analyze the operational implications for these practices in Brazilian hospitals.
\end{abstract}

Key words Reprocessing, Medical devices, Regulation, Sanitary surveillance, Operational pratices
Resumo É crescente o arsenal tecnológico utilizado nas intervenções do cuidado à saúde, ampliado continuamente com a introdução de novos produtos e artigos. Os problemas relacionados à reutilização de produtos médicos, tanto os considerados como reusáveis quanto os de uso único, abrangem aspectos de natureza técnico-operacional, econômica, ambiental, jurídica, política e ética. Este estudo tem o objetivo de contextualizar os sistemas de regulação dos produtos médicos, $e$ analisar as implicações operacionais para a prática hospitalar brasileira. Trata-se de um artigo de revisão bibliográfica, realizada sem restrição de tempo e idioma, utilizando as bases de dados da Web of Science, Bireme, Scielo e Lilacs, com descritores específicos. A contextualização dos esquemas regulatórios de produtos médicos no mundo e no Brasil e as regras básicas das normatizações existentes sobre o reprocessamento desses produtos são as fontes de subsídio que este estudo utiliza para analisar as implicações operacionais sobre essas práticas em hospitais brasileiros.

Palavras-chave Reprocessamento, Produtos médicos, Regulação, Vigilância sanitária, Práticas operacionais 


\section{Introdução}

A despeito do avanço na assistência hospitalar ter sido favorecido pelo desenvolvimento da indústria de produtos médicos, a utilização destes dispositivos trouxe também sérios riscos para o paciente usuário destes materiais, suscitando questões teórico-práticas de segurança e efetividade dos processos e de riscos reais e potenciais de aquisição de infecção relacionados com a qualidade da sua utilização e manuseio ${ }^{1-6}$. No presente artigo, utiliza-se o termo produto médico como sinônimo de dispositivo, equipamento, material e artigo médico, visando manter a mesma nomenclatura utilizada pela Agência Nacional de Vigilância Sanitária (Anvisa).

A Organização Mundial de Saúde define dispositivo médico como "qualquer instrumento, aparato, utensílio, material ou outro artigo, intencionado pelo fabricante para ser usado em humanos, com a proposta de diagnóstico, prevenção, monitoramento, tratamento ou alívio de doença ou agravo, investigação, substituição ou modificação da anatomia ou processo fisiológico ou controle de concepção e nos quais não se realiza sua ação principal dentro ou sobre o corpo humano por meios farmacológicos, imunológicos ou metabólicos"7-10.

No Brasil, acumulam-se evidências de que a população é exposta a numerosos riscos e danos evitáveis, demonstrando práticas negligentes e até criminosas de agentes econômicos e também deficiências no controle sanitário a ser exercido pelo Estado. Esta situação aponta para a necessidade de qualificação da capacidade operativa do sistema de saúde, em particular, do sistema de vigilância sanitária; e sugere que a atuação dos serviços de saúde no uso das tecnologias médicas "vem se dando em ambiente de escassa informação científica e de precária organização técnico-administrativa do sistema de controle sanitário"11.

A incorporação de tecnologias, ressalta a autora, requer avaliação da relação custo-benefício e o ônus de permanente vigilância de sua utilização, da ocorrência de eventos adversos, bem como de estratégias continuadas para promover o uso racional.

Os equipamentos e produtos médicos podem se transformar em reservatórios ou fontes de microorganismos, em decorrência de práticas inadequadas de limpeza, desinfecção e esterilização, e assim causar infecção hospitalar em pacientes expostos. O risco de infecção pode estar relacionado com a duração da hospitalização, com o tipo e a qualidade dos procedimentos in- vasivos realizados durante o internamento e também com a inadequação do reprocessamento de artigos hospitalares ${ }^{1,3,6}$.

Os produtos médicos podem ser considerados itens reusáveis ou de uso único. Os artigos reusáveis ou de uso múltiplo são bens duráveis, produzidos a partir de metais, borrachas, vidros ou tecidos. Para a reutilização segura destes materiais faz-se necessária a ação de reprocessamento, que inclui limpeza e desinfecção ou esterilização, a ser aplicada ao produto médico, com controle da qualidade em todas suas etapas de modo a garantir a segurança em sua utilização ${ }^{12,13}$. Os artigos de uso único ou descartáveis são produzidos a partir de materiais plásticos ou elastoméricos, sensíveis ao calor e adquiridos pelas instituições de saúde já previamente embalados e esterilizados. O reuso desses materiais, iniciado na década de 1970, mantém-se em prática em todo o mundo, particularmente nos EUA e no Canadá ${ }^{1,3,5,6}$.

A despeito do reuso de artigos descartáveis e da controvérsia na literatura sobre esta prática, ainda não há estudos que comprovem a segurança e a eficácia do reuso de todos esses artigos, realizado, muitas vezes, de forma indiscriminada, como visto em alguns países, a exemplo do Brasil. Adicionalmente, a literatura registra diversos surtos de infecção associados a produtos considerados de uso múltiplo ou reusáveis ${ }^{5,8,14}$, realçando a problemática do reprocessamento de produtos médicos de forma geral, independente de estes produtos serem de uso único ou reusáveis. Ratificando esta informação, verifica-se neste momento, em diferentes regióes do Brasil, surtos de infecções pós-cirúrgicas por Micobactérias de Crescimento Rápido (MCR), relacionados a falhas nos processos de limpeza, desinfecção e esterilização de produtos médicos reusáveis ${ }^{15}$.

Dentre os riscos associados ao reuso de produtos de uso único, diversos autores ${ }^{1-3,7,8}$ destacam os seguintes: infecção; contaminação do material com endotoxinas; biofilmes, presença de resíduos tóxicos dos produtos usados para a limpeza e desinfecção ou esterilização; bioimcompatibilidade com proteínas dos últimos usuários que eventualmente permaneceram no material; inconfiabilidade funcional; ausência de integridade física e de barreiras de proteção, entre outros.

Dada a relevância dos problemas relacionados com a reutilização de produtos médicos, os Estados nacionais buscam organizar seus sistemas regulatórios de modo a prevenir, eliminar ou diminuir riscos reais ou potenciais nas práticas de cuidados de saúde. Nesse sentido, este estudo tem por objetivo contextualizar os sistemas 
de regulação do reprocessamento de produtos médicos e analisar as implicações operacionais do sistema regulatório brasileiro para a prática hospitalar no país.

\section{Metodologia}

Trata-se de um estudo de revisão sistemática de dados secundários, sem restrição de período e idioma, realizado através de consultas nas bases eletrônicas do Web of Science, Pubmed, Medline e Bireme, utilizando os seguintes descritores: reprocessing device medical, reprocessing device single use, reuse device medical, regulation device materials, regulatory devices medicals. Foram excluídos os artigos que tratavam apenas dos aspectos regulatórios dos produtos médicos, sem mencionar a regulação do reprocessamento desses produtos. Assim, dos 110 artigos encontrados nas bases eletrônicas acima citadas, foram analisados 32 .

\section{Produtos médicos: \\ Políticas internacionais de classificação e regulação do reprocessamento}

Embora existam diferentes sistemas regulatórios de produtos médicos entre os países, todos aplicam a filosofia do gerenciamento de risco, e assim, o grau de escrutínio regulatório a ser imposto para qualquer produto médico deve ser proporcional ao seu risco potencial ${ }^{16-19}$. Internacionalmente, há também uma variedade de níveis regulatórios no estabelecimento de políticas de reuso de produtos médicos, que de modo geral, tendem a ter um caráter preventivo, com recomendações que objetivam a segurança da saúde pública ${ }^{18}$.

Na Austrália, o The Australian Therapeutic Goods Administration (TGA), órgão nacional regulador de produtos médicos, emite o Australian Register of Therapeutic Goods (ARTG), que é o número do produto a ser comercializado. Nesse país, o TGA permite o reuso de produtos médicos de uso único somente nas condições em que esse produto mantenha a qualidade de um equivalente original. Em dezembro de 2003, a Austrália e a Nova Zelândia assinaram um tratado para normatizar produtos terapêuticos e médicos na forma de uma agência conjunta bi-nacional, em substituição à Australian Therapeutic Goods Administration (TGA) e à New Zealand Medicines and Medical Devices Safety Authority (MEDSAFE), a fim de estabelecer um esquema regulatório conjunto para produtos médicos e medicinais, com previsão de ser operacionalizada em $2006^{8,18,20}$.

No Canadá, a licença de um produto médico é concedida pelo Therapeutic Products Directorate e ainda não existe uma norma federal regulando o reuso e reprocessamento de qualquer tipo de produto médico. No Quebec e em Manitoba existem algumas diretivas para hospitais acerca do reuso desses produtos. Entretanto, as regulações do Canada's Food and Drug Act and Medical Devices, órgão nacional responsável pelos produtos médicos, direcionam-se para fabricantes e comercializadores de produtos médicos, não objetivando o uso ou reuso desses materiais ${ }^{5,8,14,18}$.

O sistema regulatório de produtos médicos da União Européia (UE) é baseado na diretiva 2007/47/EC que designa responsabilidades do ciclo regulatório para fabricantes de produtos médicos, Estado e organizações de certificação, os chamados "corpos de certificação ou de notificação", que são organizações destinadas a realizarem auditorias de produtos e sistemas de qualidade. As Diretivas da União Européia não são direcionadas para o reuso e reprocessamento de produtos médicos, embora a prática do reprocessamento desses materiais seja frequente e heterogênea entre os países membros ${ }^{10,14}$. De modo geral, nenhuma autoridade européia possui uma política documentada embasando e assegurando o reuso de dispositivos médicos de uso único. A prática do reprocessamento desses materiais é bastante difundida através dos países membros da UE e atualmente o status regulatório legal desses países é bastante heterogêneo. Não existe nenhum nível regulatório para o reuso e reprocessamento de produtos médicos de uso único na Áustria, Bélgica, Dinamarca, Grécia, e Suíça. O reuso e reprocessamento é parcial ou indefinido em países como Noruega, Suécia, Itália, Portugal e Alemanha. O reuso é proibido na Finlândia, França, Hungria, Reino Unido e Espanha ${ }^{10,18}$.

No Reino Unido, em 2003, a agência de dispositivos médicos (The Medical Devices Agency), uniu-se à agência de controle de medicina (The Medicines Control Agency), surgindo o The Medicines and Healthcare Products Regulatory Agency (MHRA), que se constitui numa agência executiva do departamento de saúde do país, responsável pela proteção da saúde pública. O MHRA posiciona-se fortemente contra o reuso de dispositivos descartáveis e argumenta que tal prática compromete a segurança, desempenho e efetividade dos produtos e que o risco de exposição para pacientes e reprocessadores é maior do que qualquer benefício ${ }^{20}$. 
Nos EUA, o The Center for Devices and Radiological Health (CDRH), um órgão do Food and Drug Administration (FDA), é responsável pela regulação sanitária das empresas que fabricam, embalam, rotulam e/ou importam produtos médicos. Em 1999, reestruturou sua política de reutilização de dispositivos de uso único, segundo um princípio de equidade regulatória, no qual fabricantes de produtos médicos originais, empresas reprocessadoras terceirizadas e instituições hospitalares estão num mesmo patamar de controle regulatório. Ficaram excluídos desta legislação, instituições de saúde não hospitalares (clínicas, hospitais-dia, unidades de cuidados prolongados, cuidado domiciliar), dispositivos de uso único abertos, mas não usados, marca-passos de implante permanente e hemodializadores $^{1,3,8,17,19,21-26}$.

O FDA classifica os produtos médicos segundo o grau de risco que representam para a segurança do paciente, dispondo-os em três classes: classe I (risco mínimo), classe II (maior risco) e classe III (alto risco). Os produtos das classes I e II requerem notificação pré-comercialização, denominado $510 \mathrm{~K}$, que consiste num processo de autorização que demonstra que o produto médico proposto é equivalente, em segurança e efetividade, a um produto já comercializado no país. Os produtos classe III requerem uma autorização pré-comercialização, o PMA, que deve demonstrar, por evidências científicas validadas, segurança e efetividade do produto. $\mathrm{O}$ reprocessamento dos produtos médicos de uso único das classes I, II e III seguem as mesmas normativas acima descritas ${ }^{1,3,8,17,19,21-26}$.

Para o FDA, o risco à saúde associado ao reprocessamento de produtos médicos de uso único varia de acordo com o tipo de cada produto. Hospitais e empresas reprocessadoras que realizam esta prática são considerados como "fabricantes" e a submissão a esta Agência, para liberação do reprocessamento de um produto de uso único depende da classificação do produto. Deste modo, o reprocessamento de produtos de uso único classe I e II exige submissão de notificação pré-comercialização, enquanto os produtos de classe III, uma aprovação pré-comercialização. Em outubro de 2002, o FDA incluiu dados de validação dos processos de limpeza e esterilização, além dos dados que demonstram que esses produtos mantêm a funcionalidade de um produto "substancialmente equivalente", mesmo após o número máximo de vezes que o dispositivo é reprocessado, como declarado na notificação pré-comercialização. A notificação pré-comercialização e a aprovação pré-comercialização (PMA) são específicas para cada produto; deste modo, cada dispositivo a ser liberado para reprocessamento requer um $510 \mathrm{~K}$ ou um PMA 1,16,17,22-24,26.

Atualmente, os EUA apresentam o mais rigoroso controle regulatório estabelecido para as práticas de reuso e reprocessamento de produtos médicos do mundo. Entretanto, as normativas deste país, fixadas pelo FDA, tal como ocorre em outros países, são obscuras em pontos cruciais do reprocessamento, suscitando questões quanto à implementação dessas normativas, especialmente nas instituições hospitalares norteamericanas.

O sistema regulatório de produtos médicos do FDA centra-se na comercialização do produto e classifica-os de forma distinta da tradicional e consagrada categorização de produtos, diferindo da compreensão do risco relacionado ao produto utilizado num procedimento assistencial. Desta forma, um artigo considerado crítico como um cateter venoso central, de alto risco para transmissão de infecção se inadequadamente esterilizado, é classificado pelo FDA como classe II, de risco intermediário e isento da exigência do PMA. As duas submissões para pré-comercialização e/ ou reprocessamento de dispositivos médicos, o 510K e o PMA, são ambíguas em suas exigências para autorização destes processos. Por exemplo, de que maneira o fabricante/reprocessador terceirizado/instituição hospitalar, deverá comprovar que o "produto médico reprocessado classe I e II é equivalente em segurança e efetividade a um produto original, não processado", exigência para cumprimento do $510 \mathrm{~K}$ ? Quais os padrões de controle que as unidades reprocessadoras de materiais usarão para demonstrar "validade científica e evidências clínicas da segurança e efetividade do dispositivo médico de uso único reprocessado da classe III", exigido pelo PMA? Sem metodologia definida, certamente haverá diferentes experiências e ensaios clínicos para o cumprimento desta normativa. Todas as metodologias apresentadas são aceitas? Outra indefinição refere-se à aceitação, pelo FDA, de grupos similares de produtos; ou seria obrigatório que cada modelo de produto deva submeter-se ao $510 \mathrm{~K}$ e ao PMA? Esta regulação isenta outras instituições de saúde que também praticam reuso e reprocessamento de produtos médicos de uso único, a exemplo de clínicas, unidades de cuidados prolongados, como psiquiatria, hospitais-dia e cuidado domiciliar, que permanecem não reguladas. São questões em aberto, que se apresentam como limitações desse marco regulatório. 
Regulação brasileira de reprocessamento de produtos médicos e possíveis implicações operacionais para a prática hospitalar

Os artigos de uso único são objeto de preocupação do Ministério da Saúde (MS), que desde 1984, quando ocorreu uma Reunião de Peritos, vem discutindo e tentando regulamentar o reuso destes produtos no país. Em 1986, foram expedidas as Portarias MS no 03 e 04, que traziam definições e normas sobre o reuso de artigos descartáveis.

Em 2006, a Anvisa editou em 11 de agosto, a Resolução da Diretoria Colegiada - RDC no 156, que dispõe sobre o registro, rotulagem e reprocessamento de produtos médicos; a Resolução Especial - RE no 2.605, que estabelece uma relação de 66 produtos proibidos de reprocessamento no país, e a RE no 2.606, que define as diretrizes para elaboração, validação e implantação de protocolos de reprocessamento de produtos médi$\cos ^{13,27,28}$. Em julho de 2007, a Anvisa tornou a editar nova Resolução Especial, de no $2.305^{29}$, prorrogando em cento e oitenta dias o prazo para entrada em vigência da normativa estabelecida no artigo 17 da RE 2.606, demonstrando, com esta medida, a dificuldade dos hospitais brasileiros na operacionalização da regulamentação proposta de reprocessamento seguro de produtos médicos.

O maior incentivo, no mundo, para a adoção do reuso de artigos descartáveis é o econômico, que exerce, talvez, a maior influência na adoção desta prática, pela redução de custos, uma vez que tais materiais são caros, dificultando sua sustentação no sistema de saúde, seja público ou privado $^{14,30}$.

As razões de ordem técnica contrárias ao reuso destes artigos são várias, desde o simples questionamento se a matéria prima de um artigo projetado para um único uso permite ou suporta utilizações posteriores. O risco de reutilizar um material, cujo fabricante afirma que deve ser desprezado após um único uso, deveria, por si só, ser fator de cuidado redobrado e de decisão contrária ao reuso. Também há dúvidas se são garantidas as condições de segurança microbiológica, apirogenicidade, atoxicidade e funcionabilidade do produto original. Ademais, problematiza-se o próprio design destes materiais: normalmente não são desmontáveis; apresentam conformações complexas, engates, válvulas, balões, filtros, lumens reduzidos e áreas de difícil acesso e requerem conhecimento sobre o grau de especialização do produto, seu funcionamento e montagem, desafiando, dificultando ou impossibilitando o processo de limpeza, o que pode contribuir para falhas no processo de esterilização $o^{2,3,6,8}$.
Ainda em relação ao processo inicial de limpeza dos artigos descartáveis, há que se considerar que muitos produtos de limpeza agridem a matéria prima destes artigos, concorrendo para a deterioração das propriedades mecânicas dos dispositivos de uso único. Além disso, existe a questão da água utilizada para o enxágue dos artigos limpos, quanto ao número de enxágues, para remoção dos resíduos químicos empregados e à pureza suficiente para a isenção de endotoxinas - no caso de artigos que acessam o sistema circulatório - realidade distante da maioria das práticas de reprocessamento das Centrais de Material e Esterilização (CME) no Brasil.

Assim, ao se considerar o reprocessamento e o reuso de um produto descartável, faz-se necessário conhecer os possíveis riscos associados, avaliar o impacto no paciente versus a economia potencial dos custos institucionais e atentar que determinados riscos podem não ser detectáveis e/ou preveníveis. Os eventos adversos relacionados ao reprocessamento frequentemente são subinformados, em grande parte por deficiências dos sistemas de vigilância sanitária, dificuldades para determinar a real causa dos eventos e questões de responsabilidade ética que envolvam esses procedimentos $3,8,14$.

Diante destas considerações, questiona-se o que realmente deve distinguir um produto de uso único de um produto reutilizável e quais critérios a agência regulatória brasileira (Anvisa) utiliza para aceitar do fabricante o rótulo de um produto como de uso único ou de uso permanente. Para alguns autores ${ }^{6,31}$, o que define um dispositivo médico de uso único de outro reusável transcende questões econômicas ou de complexidade tecnológica e centra-se na exclusiva descrição e intenção do fabricante do produto.

Não existem padrões consensuais que diferenciem qualidade, funcionalidade ou vida útil entre produtos reusáveis ou de uso único ${ }^{31}$. O FDA especifica que a instituição ou profissionais que reusam produtos de uso único devem demonstrar que o artigo pode ser adequadamente limpo e esterilizado; que as características físicas ou a qualidade do artigo não foi afetada e permanece segura e efetiva para o uso clínico pretendido. Este critério deveria também ser aplicado igualmente para instrumentos reusáveis que são reprocessados nos hospitais.

Quanto aos aspectos de ordem legal, ainda não existe, no Brasil, legislação específica que impute ao promotor do reuso qualquer responsabilidade criminal, situação frequente nos Estados Unidos. Nesta linha de considerações, surge 
um conjunto de indagações sobre questões cruciais ainda não definidas no país, a exemplo da responsabilidade legal pela qualidade dos materiais reprocessados, sejam de uso único ou múltiplo, se as instituições hospitalares ou as empresas reprocessadoras. Se a maioria destas apenas esteriliza o material, cuja limpeza é realizada nas instituições hospitalares, como dividir estas responsabilidades? Como uma empresa terceirizada reprocessadora pode assegurar a esterilização se não realiza ou desconhece como foi realizado o processo de limpeza e enxágue do artigo a ser esterilizado? Como as instituições hospitalares estabelecem a cobrança de um artigo utilizado várias vezes?

A despeito de avanços na regulamentação brasileira sobre reprocessamento de produtos médicos, vários dispositivos das referidas Resoluções da Anvisa 156, 2.605 e 2606 estão mal formulados, contêm imprecisões e conteúdo vago, dando margem a diversificadas interpretações por parte dos serviços de saúde, empresas terceirizadas de reprocessamento e fabricantes ou importadores desses produtos.

A Resolução 156/2006 categoriza os referidos produtos como passíveis de reprocessamento e de reprocessamento proibido e estabelece que este enquadramento deve ser feito no registro do produto, quando o fabricante ou importador deve apresentar à Anvisa documentação que fundamente a indicação. No entanto, a normativa não especifica a documentação requerida e os parâmetros de avaliação para fabricantes ou importadores no ato de inscrição e registro de produtos permanentes e de uso único.

A Resolução 2.605/2006 lista 66 produtos enquadrados como de uso único e proibidos de reprocessamento, mas não explicita os critérios utilizados na escolha dos artigos que compõem a lista negativa. Tal formulação não favorece a compreensão das bases técnico-científicas da regulamentação de uma prática que envolve questões tão relevantes para a saúde no país.

A Resolução 2.606/2006 define que as empresas terceirizadas e os serviços de saúde que reprocessam artigos críticos e semicríticos devem elaborar, validar e implantar protocolos para cada marca e tipo de produto selecionado, com descrição detalhada de todos os passos do reprocessamento, além da garantia da qualidade do resultado de todas as fases, incluindo a avaliação de funcionalidade, esterilidade, rastreabilidade, condições de armazenamento e descarte de cada produto reprocessado. Normatiza também, que cada produto crítico e semicrítico a ser reprocessado, sem especificar se de uso único ou múltiplo, deve ter um prontuário com informações referentes ao artigo (dimensões, estrutura, composição), registro na Anvisa, fabricante e fornecedor, nome do responsável pelo reprocessamento, local e data de cada reprocessamento. A referida Resolução 2.606, ao tempo que exige a elaboração, validação e implantação de protocolos de reprocessamento de produtos médicos, não indica qual a metodologia aceitável para a validação dos processos a serem realizados pelos hospitais, o que não apenas dificulta a busca por sua operacionalização, mas também, dá margem à realização de protocolos de validação com resultados duvidosos, trazendo insegurança no reprocessamento de produtos. Ademais, esta regulamentação é imprecisa quando exige garantia de qualidade de todas as etapas do processo, incluindo avaliação da funcionalidade, esterilidade, pirogenicidade, atoxicidade e integridade. Novamente interroga-se qual a metodologia aceitável para esses controles de qualidade. Há que se fazer estes testes para todos os artigos críticos e semicríticos? Como, quem e quando avaliar a funcionalidade e a integridade de todos os artigos reprocessados, tendo em conta a imensidão de produtos médicos existentes em uma instituição de saúde? Qual deverá ser a periodicidade mínima destes testes? Como elaborar um prontuário para cada artigo crítico e semicrítico, com os dados exigidos por essa normativa, considerando a realidade física-funcional-organizacional da maioria das CMEs dos hospitais do Brasil e a vastidão de produtos que compõem o seu arsenal?

Análise recente da regulamentação da Anvisa sobre reprocessamento de produtos médicos aponta doze nós críticos, a exemplo da baixa sensibilidade de critérios para a definição de produto de uso único, ausência de transparência de critérios utilizados na elaboração da lista negativa de produtos e sua potencial obsolescência, entre outros, que dificultam a operacionalização dessas normas ${ }^{32}$. Segundo esta análise, a lista negativa de produtos da Resolução 2605 desvia a atenção do risco real associado aos processos de limpeza, desinfecção, esterilização e seus controles de qualidade, para um risco potencial de "o que pode e o que não pode ser reprocessado”, minimizando a problemática do processo de trabalho das CMEs do país, cerne da segurança de qualquer produto a ser reprocessado.

Diante do exposto, questiona-se como lidar com a incerteza científica associada ao reprocessamento de um artigo, seja de uso único ou reprocessável, sem as condições básicas ditas pela 
literatura, que possam garantir a segurança dos processos? Que estratégias devem ser adotadas pelos gerentes de hospitais para a realização segura do reprocessamento de produtos médicos, sejam descartáveis ou reusáveis? Como validar os protocolos de reprocessamento nas CMEs das unidades de saúde de um país com tanta diversidade e especificidades como o nosso, sem uma diretriz que contemple estas diferenças? Como monitorar/fiscalizar/controlar estes processos nas inúmeras unidades de saúde do país? Os profissionais de vigilância sanitária, que devem fiscalizar o cumprimento das normas sanitárias, têm condições de realizar o controle desta atividade?

Trata-se de relevante problema de saúde pública do âmbito da vigilância sanitária, que é definida na Lei Orgânica da Saúde (no 8.080/1990), como "um conjunto de ações capaz de eliminar, diminuir ou prevenir riscos à saúde e de intervir nos problemas sanitários decorrentes do meio ambiente, da produção e circulação de bens e da prestação de serviços de interesse da saúde". Cabelhe, portanto, a tarefa não apenas de normatizar, mas também de fiscalizar o cumprimento das normas que visam a proteção da saúde.

Sem a devida fiscalização, a regulamentação do reprocessamento de produto médicos, editada desde 2006, vem sendo retardada em sua implementação nos hospitais brasileiros. Além das questões que suscita, tal retardo desafia a própria

\section{Referências}

1. Smith JJ, Henderson JA, Baim DS. The Food and Drug Administration and Reprocessing of SingleUse Medical Devices: a revised policy and new questions. J. Vasc. Interv Radiol 2002; 13(12):1179-1182.

2. Graziano KU, Balsamo AC, Lopes CLBC, Zotelii MFM, Couto AT, Paschoal MLH. Critérios para avaliação das dificuldades na limpeza de artigos de uso único. Rev Latino-am Enfermagem 2006; 14(1):70-76.

3. United States General Accounting Office (USGAO). Health, Education and Human Services Division. Single-Use Medical Devices. Little Available Evidence of Harm From Reuse, but Oversigth Warrented. Report to Congressional Requesters. Washington, D.C.: USGAO; 2000. p. 205-248.

4. Altenstetter C. EU and Member State Medical Devices Regulation. International Journal of Technology Assessment in Health Care 2003; 19(1):228-248.

5. Health Canada. Therapeutic Products Directorate Health Cross. Tower B, Ottawa, Ontário: Health Canada; 2004. legitimidade da norma, reafirmando a problemática que envolve a reutilização de produtos médicos, tanto os reusáveis, quanto os considerados de uso único. Talvez uma metodologia alternativa seja a de desenvolver um sistema regulatório de controle de processo em lugar do atual controle do produto; e enquanto proposições assertivas não são vislumbradas neste cenário, permanece o desafio sobre como operacionalizar efetivamente a atual política regulatória de reprocessamento de produtos médicos no Brasil, de modo a reduzir os riscos e conferir segurança e qualidade nos cuidados em saúde. Parafraseando Marx "os filósofos têm somente interpretado o mundo: trata-se, porém, de transformá-lo".

\section{Colaboradores}

EAM Costa e EA Costa participaram igualmente de todas as etapas de elaboração do artigo.

6. Greene VW. Reuse of medical devices labeled for single-use. In: Mayhall GC, editor. Hospital Epidemiology and Infection Control. Baltimore: Williams \& Wilkins; 2004. p. 1535-1545.

7. Bright J. European medical device regulatory Law and product liability. Journal of Hospital Infection 1999; 43(Supl.):169-175.

8. World Health Organization (WHO). Medical Device Regulations. Global overview and guiding principles. Geneva: World Health Organization (WHO); 2003.

9. The Global Harmonization Task Force (GHTF). Information Document Concerning the Definition of the term Medical Device. GHTF; 2005.

10. The European Parliament and The Council of The European Union. Directive 2007/47/EC of the Parliament and of the Council. Official Journal of the European Union 2007. 
11. Costa EA. Vigilância Sanitária: Proteção e defesa da saúde. In: Almeida N, Rouquayrol MZ, organizadores. Epidemiologia \& Saúde. 6a ed. Rio de Janeiro: Guanabara Koogan; 2003. p. 357-387.

12. Sehulster LM, Schultz JK. Central Sterile Supply. In: Mayhall GC, editor. Hospital Epidemiology and Infection Control. Baltimore: Williams \& Wilkins; 2004. p. 1301-1310.

13. Agência Nacional de Vigilância Sanitária. Resolução RDC No. 156, de 11 de agosto de 2006. Dispõe sobre registro, rotulagem e reprocessamento de produtos médicos e dá outras providências. Brasília: Diário Oficial da União 2006; 15 fev.

14. Cookson R, Hutton J. Regulating the economic evalution of pharmaceuticals and medical devices: a European perspective. Health Policy 2003; (63):167178.

15. Agência Nacional de Vigilância Sanitária (Anvisa). Notícias da Anvisa. Micobactérias: medidas mais rigorosas para prevenir infecções. Brasília: 2009; 2 de março. [acessado 2009 mar 10]. Disponível em: http://www.anvisa.gov.br/divulga/noticias/2009/ 020309.htm.

16. Drum B. FD. A regulation of labeling and promotional claims in therapeutic color vision devices: A Tutorial. Neuroscience. Cambridge University Press 2004; (21):461-463.

17. Foy JR, Bush B. Orthopaedic Joint devices: The FDA's short answer to your questions. J Am Acad Orthop Surg 2008; 16(Supl. 1):S123-S128.

18. Day P. What is the evidence on the safety and effectiveness of the reuse of medical devices labelled as single-use only? New Zealand Helath Tecnology Assessement (NZHTA). Tech Brief Series 2004; 3(2). [acessado 2009 ago 12]. Disponível em: http:// nzhta.chmeds.ac.nz/medical_devices.pdf

19. Mansfield E, O'leary TJ, Gutman SI. Food and Drug Administration Regulation of in vitro diagnostic devices. J Mol Diagn 2005; 7(1):2-7.

20. Ghost H, Dilip S, Margot F, Lynnette R. The role of the therapeutic goods administration and medicine and Medical Devices Safety Authority in evaluating complementary and alternative medicines in Australia and New Zealand. Toxicology 2006; 221(1):8894.

21. Feigal DW. Testimony on Reuse of Medical Devices. Food and Drug Administration. June, 2000. [acessado 2011 out 18]. Disponível em: http://www.fda.gov/ NewsEvents/Testimony/ucm114926.htm

22. Food and Drug Administration(FDA). Department of Health and Human Services. Center for Devices and Radiological Health. Issues Final Guidance on reuse of single-use medical devices. USA: Food and Drug Administration(FDA); 2000.

23. Food and Drug Administration (FDA). Department of Health and Human Services. Center for Devices and Radiological Health. Enforcement Priorities for single-use devices reprocessed by third parties and hospitals. USA: Food and Drug Administration (FDA); 2000.
24. Food and Drug Administration (FDA). Department of Health and Human Services. Center for Devices and Radiological Health. Reuse of single-use devices. Featurettes/ News to use. USA: Food and Drug Administration(FDA); 2000.

25. Food and Drug Administration (FDA). Department of Health and Human Services. Center for Devices and Radiological Health. Labeling recommendations for single-use devices reprocessed by third parties and hospitals. Final Guidance for Industry and FDA. USA: Food and Drug Administration(FDA); July, 2001.

26. Smith JJ, Henderson JA. FDA regulation of implantable sensors demonstrating safety and effectiveness for marketing in the US. IEEE Sensors Journal 2008; 8(1):52-56.

27. Agência Nacional de Vigilância Sanitária (Anvisa). Resolução RE No. 2.605, de 11 de Agosto de 2006. Contém a lista de produtos que não podem ser reprocessados. Diário Oficial da União 2006; 15 fev.

28. Agência Nacional de Vigilância Sanitária (Anvisa). Resolução RE No. 2.606, de 11 de Agosto de 2006. Dispõe sobre as diretrizes para elaboração, validação e implantação de protocolos de reprocessamento de produtos médicos e dá outras providências. Diário Oficial da União 2006; 15 fev.

29. Agência Nacional de Vigilância Sanitária (Anvisa). Resolução RE No. 2.305, de 31 de Julho de 2007. Prorroga em cento e oitenta dias o prazo estabelecido no Art. 17 da RE 2606/2006. Diário Oficial da União 2007; 15 fev.

30. Padoveze CM. Riscos associados ao Reprocessamento de Artigos de Uso Único. In: Associação Paulista de Estudos e Controle de Infecção Hospitalar (APECIH), organizador. Reprocessamento de Artigos de Uso Único. $1^{\text {a }}$ ed. São Paulo: Associação Paulista de Estudos e Controle de Infecção Hospitalar (APECIH); 2008.

31. Stoermer WB. Reprocessing single-use devices: why does the debate continues? Medical Device \& Diagnostic Industry Magazine 1999. [acessado 2011 out 18]. Disponível em: http://www.mddionline. com/article/reprocessing-single-use-devices-whydoes-debate-continue

32. Graziano KU. Análise da legislação atual sobre reprocessamento de artigos de uso único. In: Associação Paulista de Estudos e Controle de Infecção Hospitalar (APECIH), organizador. Reprocessamento de Artigos de Uso Único. $1^{\text {a }}$ ed. São Paulo: Associação Paulista de Estudos e Controle de Infecção Hospitalar (APECIH); 2008.

Artigo apresentado em 22/09/2009

Aprovado em 27/01/2010

Versão final apresentada em 10/02/2010 